\title{
Neutralino with the Right Cold Dark Matter Abundance in (Almost) Any Supersymmetric Model
}

\author{
Graciela Gelmini ${ }^{1}$ and Paolo Gondolo ${ }^{2}$ \\ ${ }^{1}$ Department of Physics and Astronomy, UCLA, 475 Portola Plaza, Los Angeles, CA 90095, USA \\ ${ }^{2}$ Department of Physics, University of Utah, 115 S 1400 E \# 201, Salt Lake City, UT 84112, USA \\ gelmini@physics.ucla.edu, paolo@physics.utah.edu
}

\begin{abstract}
We consider non-standard cosmological models in which the late decay of a scalar field $\phi$ reheats the Universe to a low reheating temperature, between $5 \mathrm{MeV}$ and the standard freeze-out temperature of neutralinos of mass $m_{\chi}$. We point out that in these models all neutralinos with standard density $\Omega_{\text {std }} \gtrsim 10^{-5}\left(100 \mathrm{GeV} / m_{\chi}\right)$ can have the density of cold dark matter, provided the right combination of the following two parameters can be achieved in the high energy theory: the reheating temperature, and the ratio of the number of neutralinos produced per $\phi$ decay over the $\phi$ field mass. We present the ranges of these parameters where a combination of thermal and non-thermal neutralino production leads to the desired density, as functions of $\Omega_{\text {std }}$ and $m_{\chi}$.

PACS numbers: 14.60.St, $98.80 . \mathrm{Cq}$
\end{abstract}

In supersymmetric models, the lightest supersymmetric particle (LSP), usually a neutralino $\chi$, is a good cold dark matter candidate. The cosmological density of the neutralino is a function of the supersymmetric model parameters, and it has been computed theoretically to high precision. Requiring the LSP to have the measured dark matter density constrains the models considerably to very narrow regions in parameter space.

The standard computation of the relic density relies on the assumptions that the entropy of matter and radiation is conserved, that neutralinos are produced thermally and were in thermal and chemical equilibrium before they decoupled. The decoupling, or freeze-out, temperature, i.e. the temperature after which their number practically does not change, is $T_{\text {f.o. }} \simeq m_{\chi} / 20$, where $m_{\chi}$ is the neutralino mass. The standard neutralino relic density $\Omega_{\text {std }}$ obtained in this way can be larger or smaller than the measured density of cold dark matter $\Omega_{\mathrm{cdm}}=0.113 \pm 0.009 h^{-2}[\underline{1}]$.

However, there are non-standard cosmological models in which the assumptions mentioned above do not hold. These include models with moduli decay [2], Q-ball decay [3], and thermal inflation [4]. In all of these models there is a late episode of entropy production and non-thermal production of the LSP in particle decays is possible.

We concentrate on cosmological models in which the late decay of a scalar field $\phi$ reheats the Universe to a low reheating temperature $T_{\mathrm{RH}}$, which can be smaller than $T_{\text {f.o. }}$ without spoiling primordial nucleosynthesis $[5]$ $\left(5 \mathrm{MeV} \lesssim T_{\mathrm{RH}} \lesssim T_{\text {f.o. }}\right)$. Such scalar fields are common in superstring models where they appear as moduli fields. The decay of $\phi$ into radiation increases the entropy, diluting the neutralino number density. The decay of $\phi$ into supersymmetric particles, which eventually decay into neutralinos, increases the neutralino number density. We denote by $b$ the net number of neutralinos produced on average per $\phi$ decay.

Both thermal and non thermal production mechanisms have been discussed in the literature $2,6,7,6,6,10$, 11, 12, 13]. Several supersymmetric models with particular combinations of $T_{\mathrm{RH}}, b$, and $m_{\phi}$ have been studied [2, 7, 8, 9, 10, 11, 12, 13]. Ref. 7] assumed that one neutralino is produced per $\phi$ decay $(b \simeq 1)$, and concluded that in the MSSM the non-thermal neutralino production leads to an overabundance of relic neutralinos by several orders of magnitude. Also the thermalization of $\chi$ 's produced in decays was discussed in Ref. 7]. Refs. [8, 9, 12] studied the thermal production of LSPs during the decay of a scalar field with $b=0$, and mentioned the possibility of $b \neq 0$ but did not study it. Ref. 2] considered purely non-thermal production. Ref. 13] is closest to our work, in that both thermal and non-thermal production were considered, but the general strategy to rescue models with too low or too high standard relic density remained, in our opinion, unclear.

In spite of all the above work, no coherent overview of the issue, which is remarkably easy to understand, has to our knowledge been laid down. This is what we intend to provide in this letter.

Let $\Omega_{\text {std }}$ be the density that neutralinos in a particular model would have with the usually assumed cosmology. An appropriate combination of the following two parameters can bring the relic neutralino density to the desired value $\Omega_{\mathrm{cdm}}$ : the ratio $b / m_{\phi}$ giving the average number of LSPs produced per unit of energy released in each $\phi$ decay and the reheating temperature $T_{\mathrm{RH}}$ (which must be $\left.<T_{\text {f.o. }}\right)$. We assume that the oscillating $\phi$ field dominates the energy density of the Universe at early times and that at $T_{\mathrm{RH}}$ the Universe becomes dominated by the radiation produced in $\phi$ decays. We follow the usual choice of the parameter $T_{\mathrm{RH}}$ as the temperature the Universe would attain under the assumption that the $\phi$ decay and subsequent thermalization are instantaneous

$$
\Gamma_{\phi}=H_{\text {decay }}=\left(\frac{8 \pi}{3}\right) \rho_{R}=\sqrt{\frac{8}{90} \pi^{3} g_{\star}} \frac{T_{\mathrm{RH}}^{2}}{M_{P}} .
$$


$\Gamma_{\phi}$ is the decay width of the $\phi$ field,

$$
\Gamma_{\phi} \simeq \frac{m_{\phi}^{3}}{\Lambda_{\text {eff }}^{2}} .
$$

If $\phi$ has non-suppressed gravitational couplings, as is usually the case for moduli fields, the effective energy scale $\Lambda_{\text {eff }} \simeq M_{P}$. In models with intermediate scales, $\Lambda_{\text {eff }}$ could be smaller [1]. Thus, with $g_{\star} \simeq 10$

$$
T_{\mathrm{RH}} \simeq 10 \mathrm{MeV}\left(\frac{m_{\phi}}{100 \mathrm{TeV}}\right)^{3 / 2}\left(\frac{M_{P}}{\Lambda_{\text {eff }}}\right) .
$$

In order not to disrupt the predictions of Big Bang nucleosynthesis, $T_{\mathrm{RH}} \gtrsim 5 \mathrm{MeV}[5]$. Thus

$$
m_{\phi} \gtrsim 100 \mathrm{TeV}\left(\frac{\Lambda_{\mathrm{eff}}}{M_{P}}\right)^{2 / 3} .
$$

The number $b$ of neutralinos produced per $\phi$-decay is highly model-dependent. It is determined by the physics of the hidden sector, by the mechanism of supersymmetry breaking, and in superstring-inspired models by the compactification mechanism.

The coupling of the $\phi$ to the gravitino arises from the term $e^{K / 2} \bar{\psi}_{\mu} \sigma^{\mu \nu} \psi_{\nu}$, where $K$ is the Kähler potential. If $m_{\phi}$ is larger than twice the gravitino mass $m_{3 / 2}$, the decay mode $\phi \rightarrow \psi_{3 / 2} \psi_{3 / 2}$ of the moduli field into two gravitinos is present with branching ratio of order 0.01 (see Refs. 14], which correct previous claims [15] that this branching would be chirally suppressed by a factor $\left.\left(m_{3 / 2} / m_{\phi}\right)^{2}\right)$. Gravitinos must then decay rapidly not to disrupt nucleosynthesis (so $m_{3 / 2} \gtrsim 100 \mathrm{TeV}$ ), and they produce comparable amounts of normal particles and their supersymmetric partners. If $m_{\phi} \gg m_{3 / 2}$, the gravitino decays during the radiation dominated epoch after the decay of the $\phi$ field (here we do not address this case and we focus on neutralino production during $\phi$ domination). When $m_{\phi}$ and $m_{3 / 2}$ are of the same order of magnitude, we can consider the gravitino decay as part of the $\phi$ decay, since they happen almost simultaneously. In this case, depending on how important the direct decay of $\phi$ into supersymmetric particles other than the $\psi_{3 / 2}$ is, $b$ can typically be $0.01-1$, but not smaller.

If instead $m_{\phi}<2 m_{3 / 2}$ more possibilities open up. The yield per $\phi$ decay $b$ can still be of order one but it can also be much smaller.

Supergravity models with chiral superfields $\Phi_{I}$ are specified in terms of the Kähler potential $K\left(\Phi_{I}, \bar{\Phi}_{I}\right)$, the superpotential $W\left(\Phi_{I}\right)$, and the gauge kinetic function $f_{\alpha \beta}\left(\Phi_{I}\right)$. Specific relations between the $\phi$ mass $m_{\phi}$, the gravitino mass $m_{3 / 2}$, and the gaugino mass $m_{1 / 2}$ arise as a consequence of the relations $m_{3 / 2}=$ $\left\langle e^{K / 2} W\right\rangle, m_{1 / 2}=\left\langle F^{J} \partial_{J} \ln \operatorname{Re} f\right\rangle$, and $m_{\phi}=\left\langle\partial^{2} V / \partial \phi^{2}\right\rangle$. With appropriate choices of $K, W$, and $f$, the hierarchy $m_{3 / 2} \gtrsim m_{\phi} \gg m_{1 / 2}$ may be achieved. Here $V=K_{I \bar{J}} F^{I} \bar{F}^{\bar{J}}-3 e^{K}|W|^{2}+\frac{1}{2}(\operatorname{Re} f)_{\alpha \beta}^{-1} D^{\alpha} D^{\beta}$ is the scalar potential, $F^{I}=-e^{K / 2} K^{I \bar{J}} D_{\bar{J}} \bar{W}$ is the $\mathrm{F}$-term of the

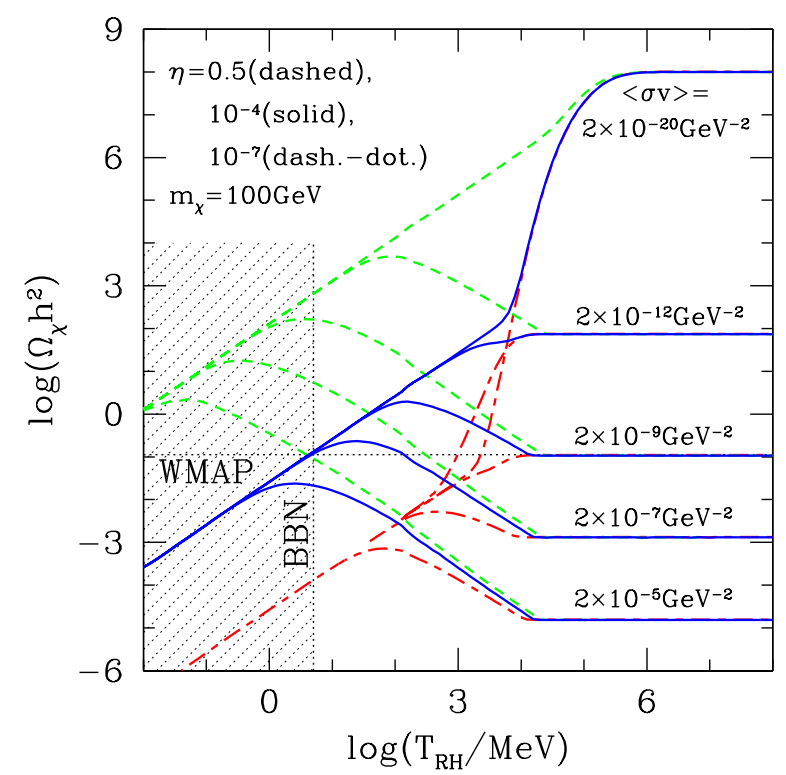

FIG. 1: Neutralino density $\Omega_{\chi} h^{2}$ as a function of the reheating temperature $T_{\mathrm{RH}}$ for illustrative values of the ratio $\eta=b\left(100 \mathrm{TeV} / m_{\phi}\right)$ (the number of neutralinos produced per unit of energy released in $\phi$ decay, in units of $\left.(100 \mathrm{TeV})^{-1}\right)$. The curves are insensitive to the initial conditions, i.e. to the value $H_{I}$ of the Hubble parameter at the beginning of the $\phi$ dominated epoch.

chiral superfield $\Phi_{I}, D^{\alpha}=T^{\alpha I J} \phi_{j} D_{I} W / W$ is the Dterm of the vector superfield, $D_{I} \mathcal{F}=\partial_{I} \mathcal{F}+\mathcal{F} \partial_{I} K$ is the Kähler covariant derivative, $\partial_{I} \mathcal{F}=\partial \mathcal{F} / \partial \Phi_{I}$, and square brackets denote vacuum values. As far as $b$ is concerned, one finds, for example, $b \simeq O(1)$ when the main $\phi$ decay mode is through a coupling of the type $h \phi \psi^{2}$ with a chiral matter supermultiplet $\psi$ in the superpotential $W$. This leads to comparable decay rates of $\phi$ into the scalar and fermionic components of $\psi$ (which are supersymmetric partners). On the other hand, it is possible that the $\phi$ field decays mostly into Higgs fields, or gauge fields (W's, Z's, photons, gluinos). In this case $b$ can be very small $10^{-2}, 10^{-4}, 10^{-6}$ etc. [2, 10]. For example, the coupling of $\phi$ to the gauge bosons arises from the term $\operatorname{Re} f_{\alpha \beta} F_{\mu \nu}^{\alpha} F^{\mu \nu \beta}$ and with non-minimal kinetic terms $f_{\alpha \beta}$ may contain $\phi$. The $\phi$ decay width into gauge bosons is then $\Gamma_{g} \sim \lambda_{g} m_{\phi}^{3} / M_{P}^{2}$ with $\lambda_{g}=\frac{\partial}{\partial \phi} \ln \operatorname{Re} f$, while that into gauginos is $\Gamma_{\tilde{g}} \sim \lambda_{\tilde{g}}^{2} m_{\phi} / M_{P}^{2}$ with $\lambda_{\tilde{g}}=$ $m_{1 / 2} \frac{\partial}{\partial \phi} \ln \left(F^{\phi} \frac{\partial}{\partial \phi} \operatorname{Re} f\right)$. Thus in principle the gaugino coupling may be suppressed relative to the coupling to gauge bosons.

Neutralinos are in kinetic equilibrium with the radiation when their scattering rate off relativistic particles is faster than the Hubble expansion rate, $\Gamma^{\text {scatt }} \gtrsim H$. During the epoch in which the Universe is dominated by the decaying $\phi$ field, $H$ is proportional to $T^{4}$ [16]. In fact, in the evolution equation for the radiation energy density (Eq. (5) below with $p=\rho / 3$ ) substitute $\rho \simeq T^{4}$ and $\rho_{\phi} \simeq M_{P}^{2} H^{2}$. Then use $H \sim t^{-1}$, write $T \propto t^{\alpha}$, match the powers of $t$ in all terms, and determine 
$\alpha=-(1 / 4)$. Hence, during the oscillating $\phi$ dominated epoch $H \propto t^{-1} \propto T^{4}$ (and $\rho_{\phi} \propto H^{2} \propto T^{8}$ ). Since $H$ equals $T_{\mathrm{RH}}^{2} / M_{P}$ at $T=T_{\mathrm{RH}}$, it is $H \simeq T^{4} /\left(T_{\mathrm{RH}}^{2} M_{P}\right)$. Since $\Gamma^{\text {scatt }} \simeq n_{\gamma} \sigma_{\text {scatt }} \simeq T^{3} \sigma_{\text {scatt }}$, we see that kinetic equilibrium is maintained at low temperatures, $T \lesssim$ $T_{\mathrm{RH}}^{2} M_{P} \sigma_{\text {scatt }}$. This is contrary to the usual radiationdominated scenario in which the temperature has to be large enough for kinetic equilibrium to be maintained. The origin of this difference is the strong $T^{4}$ dependence of $H$ on the radiation temperature. Using the known relation $\Omega_{\text {std }} h^{2} \simeq 10^{-10} \mathrm{GeV}^{-2} /\langle\sigma v\rangle$, and taking $\sigma_{\text {scatt }}$ of the same order of magnitude as the annihilation cross section $\langle\sigma v\rangle$, gives $T \lesssim\left(10^{6} \mathrm{MeV} / \Omega_{\mathrm{std}} h^{2}\right)\left(T_{\mathrm{RH}} / \mathrm{MeV}\right)^{2}$. For the following we need to assume kinetic equilibrium before neutralino production ceases: the right hand side is larger than $T_{\mathrm{RH}}$ for $\Omega_{\text {std }} h^{2} \lesssim 10^{6}\left(T_{\mathrm{RH}} / \mathrm{MeV}\right)$, so we will safely assume that kinetic equilibrium is reached.

In kinetic equilibrium the equations which describe the evolution of the Universe are:

$$
\begin{aligned}
\dot{\rho} & =-3 H(\rho+p)+\Gamma_{\phi} \rho_{\phi} \\
\dot{n}_{\chi} & =-3 H n_{\chi}-\langle\sigma v\rangle\left(n_{\chi}^{2}-n_{\chi, \mathrm{eq}}^{2}\right)+\frac{b}{m_{\phi}} \Gamma_{\phi} \rho_{\phi} \\
\dot{\rho}_{\phi} & =-3 H \rho_{\phi}-\Gamma_{\phi} \rho_{\phi} \\
H^{2} & =\frac{8 \pi}{3 M_{P}^{2}}\left(\rho+\rho_{\phi}\right) .
\end{aligned}
$$

Notice that the equations, and thus the results, depend only on the combination $b / m_{\phi}$ and not on $b$ and $m_{\phi}$ separately. In Eqs. (58), a dot indicates a time derivative, $\rho_{\phi}$ is the energy density in the $\phi$ field, which is assumed to behave like non-relativistic matter; $\rho$ and $p$ are the total energy density and pressure of matter and radiation at temperature $T$, which are assumed to be in kinetic but not necessarily chemical equilibrium; $n_{\chi}$ is the number density of LSPs, and $n_{\chi, \text { eq }}$ is its value in chemical equilibrium; finally, $H=\dot{a} / a$ is the Hubble parameter, with $a$ the scale factor. For convenience in the numerical calculations, we used as independent variable $\ln a$ and as dependent variables $Y=n_{\chi} / s$, $Y_{\phi}=\rho_{\phi} /\left(m_{\phi} s\right)$ and $T$, where $s=\left(\rho+p-m_{\chi} n_{\chi}\right) / T$ is the entropy density of the matter and radiation. We also used the first principle of thermodynamics in the form $d\left(\rho a^{3}\right)+d\left(\rho_{\phi} a^{3}\right)+p d a^{3}=T d\left(s a^{3}\right)$ to rewrite Eq. (5) as

$$
\dot{s}=-3 H s+\frac{\Gamma_{\phi} \rho_{\phi}}{T} .
$$

Initial conditions are specified through the value $H_{I}$ of the Hubble parameter at the beginning of the $\phi$ dominated epoch. This amounts to giving the initial energy density $\rho_{\phi, I}$ in the $\phi$ field, or equivalently the maximum temperature of the radiation $T_{\mathrm{MAX}}$. Indeed, one has $H_{I} \simeq \rho_{\phi, I}^{1 / 2} / M_{P} \simeq T_{\mathrm{MAX}}^{4} /\left(T_{\mathrm{RH}}^{2} M_{P}\right)$. The latter relation can be derived from $\rho_{\phi} \simeq T^{8} / T_{\mathrm{RH}}^{4}$ and the consideration that the maximum energy in the radiation equals the initial (maximum) energy $\rho_{\phi, I}$.

If the neutralino reaches chemical equilibrium, it is clear that its final density does not depend on the ini- tial conditions. An approximate condition for reaching chemical equilibrium is [9] $\langle\sigma v\rangle \gtrsim 10^{-9} \mathrm{GeV}^{-2}$ $\left(m_{\chi} / 100 \mathrm{GeV}\right)\left(T_{\mathrm{RH}} / \mathrm{MeV}\right)^{-2}$. Even without reaching chemical equilibrium, the neutralino density is insensitive to the initial conditions provided the maximum temperature of the radiation $T_{\mathrm{MAX}} \gtrsim m_{\chi}[9]$.

In Fig. 1 we show how the neutralino density $\Omega_{\chi} h^{2}$ depends on $T_{\mathrm{RH}}$ for illustrative values of the parameter $\eta=b\left(100 \mathrm{TeV} / m_{\phi}\right)$, both for neutralinos which are underdense and which are overdense in usual cosmologies.

The behavior of the relic density as a function of $T_{\mathrm{RH}}$ is easy to understand physically. If $\langle\sigma v\rangle$ is large enough so that chemical equilibrium is achieved, the usual thermal production scenario occurs for $T_{\mathrm{RH}}>T_{\text {f.o. }}$. Neutralino annihilation compensates thermal production until the latter ceases to be effective at $T=T_{\text {f.o. }}$. The LSP density is then determined by the condition $\Gamma^{\mathrm{ann}}=n\langle\sigma v\rangle \simeq H$ at $T=T_{\text {f.o. }}$. Using $H \simeq T^{2} / M_{P}$, as appropriate for a radiation dominated Universe, this leads to $Y_{0} \simeq$ $Y_{\text {f.o. }} \simeq(n / s)_{\text {f.o. }} \simeq(H / s\langle\sigma v\rangle)_{\text {f.o. }} \simeq 1 /\left(T_{\text {f.o. }} M_{P}\langle\sigma v\rangle\right)$. This gives the usual result $\Omega_{\text {std }} \simeq\left(m s_{0} / \rho_{c}\right) Y_{0} \simeq$ $\left(m s_{0}\right) /\left(\rho_{c} T_{\text {f.o. }} M_{P}\langle\sigma v\rangle\right) \simeq 2 \times 10^{-10} \mathrm{GeV}^{-2} /\langle\sigma v\rangle$. Here we used $s_{0} / \rho_{c}=2.8 \times 10^{8} \mathrm{GeV}^{-1}$. When $\langle\sigma v\rangle$ is too small for chemical equilibrium to be achieved, the usual equation does not hold. Notice that this is the case for the smallest $\langle\sigma v\rangle$ in Fig. 1.

For $T_{\mathrm{RH}}<T_{\text {f.o. }}$, there are four different ways in which the density $\Omega h^{2}$ depends on $T_{\mathrm{RH}}$ :

(1) Thermal production without chemical equilibrium. In this case $\Omega_{\chi} \propto T_{\mathrm{RH}}^{7}$ (e.g. steepest part of the $\eta=10^{-7}$, $\langle\sigma v\rangle=2 \times 10^{-20} \mathrm{GeV}^{-2}$ line). The relic density was estimated in Ref. [8]:

$$
\frac{\Omega_{\chi}}{\Omega_{\mathrm{cdm}}} \simeq \frac{\langle\sigma v\rangle}{10^{-16} \mathrm{GeV}^{-2}}\left(\frac{100 \mathrm{GeV}}{m_{\chi}}\right)^{5}\left(\frac{T_{\mathrm{RH}}}{\mathrm{GeV}}\right)^{7}\left(\frac{10}{g_{\star}}\right)^{3 / 2} .
$$

This matches our numerical calculation for $\langle\sigma v\rangle=2 \times$ $10^{-20} \mathrm{GeV}^{-2}$.

(2) Thermal production with chemical equilibrium. In this case $\Omega_{\chi} \propto T_{\mathrm{RH}}^{4}$ (e.g. steepest part of the $\eta=10^{-7}$, $\langle\sigma v\rangle=2 \times 10^{-12} \mathrm{GeV}^{-2}$ line). The neutralino freezes out while the universe is dominated by the $\phi$ field. Its freeze-out density is larger than usual, but it is diluted by entropy production from $\phi$ decays. The new freeze-out temperature $T_{\mathrm{f} . \mathrm{o}}^{\mathrm{NEW}}$ is determined by solving $n\langle\sigma v\rangle \simeq H$ at $T=T_{\text {f.o. }}^{\mathrm{NEW}}$. Using the relations between $H, a$, and $T$ in the decaying- $\phi$ dominated Universe, one finds 9 , [16] $\Omega_{\chi} \simeq T_{\mathrm{RH}}^{3} T_{\text {f.o. }}\left(T_{\mathrm{f} . \mathrm{o} .}^{\mathrm{NEW}}\right)^{-4} \Omega_{\mathrm{std}}$. Our numerical results indicate a slope closer to $T_{\mathrm{RH}}^{4}$, perhaps due to the change in $T_{\text {f.o. }}$.

(3) Non-thermal production without chemical equilibrium. Here $\Omega_{\chi} \propto T_{\mathrm{RH}}$ (e.g. leftmost part of each line). Non-thermal production is not compensated by annihilation. The production of neutralinos is purely nonthermal and the relic density depends on $\eta$. It can be estimated analytically as follows. For each superpartner produced, at least one LSP will remain at the end of a chain of decays (due to $R$-parity conservation), and thus 
$n_{\chi} \simeq b n_{\phi}$. Here $n_{\phi}=\rho_{\phi} / m_{\phi}$. At the time of $\phi$-decay $\rho_{\chi} \simeq m_{\chi} b \rho_{\phi} / m_{\phi} \simeq T_{\mathrm{RH}}^{4}$, and the entropy is $s \simeq T_{\mathrm{RH}}^{3}$. Hence $\rho_{\phi} / s \simeq T_{\mathrm{RH}}$ and $Y_{0}=Y_{\text {decay }} \simeq b T_{\mathrm{RH}} / m_{\phi}$. It follows that,

$$
\frac{\Omega_{\chi}}{\Omega_{\mathrm{cdm}}} \simeq 2 \times 10^{3} \eta\left(\frac{m_{\chi}}{100 \mathrm{GeV}}\right)\left(\frac{T_{\mathrm{RH}}}{\mathrm{MeV}}\right)
$$

(4) Non-thermal production with chemical equilibrium. In this case $\Omega \propto T_{\mathrm{RH}}^{-1}$ (e.g. central part of the $\eta=0.5,\langle\sigma v\rangle=2 \times 10^{-5} \mathrm{GeV}^{-2}$ line). Here annihilation compensates for the non-thermal production of neutralinos until the non-thermal production ceases at $T=T_{\mathrm{RH}}$. The condition for determining the relic density is $\Gamma^{\text {ann }} \simeq \Gamma_{\phi}$ at $T=T_{\mathrm{RH}}$. This leads to $Y_{0} \simeq Y_{\mathrm{RH}} \simeq \Gamma_{\phi} /\left(s_{\mathrm{RH}}\langle\sigma v\rangle\right) \simeq 1 /\left(T_{\mathrm{RH}} M_{P}\langle\sigma v\rangle\right)$. From here it follows that

$$
\Omega_{\chi} \simeq\left(T_{\text {f.o. }} / T_{\mathrm{RH}}\right) \Omega_{\mathrm{std}}
$$

With the help of Fig. 1 and formulas (11) and (12), we can separate the different ranges of $\eta$. Notice that in Fig. $1 m_{\chi}=100 \mathrm{GeV}$, thus $T_{\text {f.o. }} \simeq m_{\chi} / 20 \simeq 5 \mathrm{GeV}$. For $T_{\mathrm{RH}}>T_{\mathrm{f} \text {.o. }}$, the standard production mechanism is recovered, thus $\Omega_{\chi}=\Omega_{\text {std }}$ (indicated by the horizontal lines on the right of the Figure). If $T_{\mathrm{RH}}<T_{\mathrm{f} \text {.o. }}$, the value of $\Omega h^{2}$ depends on $\eta$. Overdense neutralinos, i.e. those with $\Omega_{\text {std }}>\Omega_{\text {cdm }}$ (above the dotted line labelled WMAP), require values of $\eta \lesssim 10^{-4}\left(100 \mathrm{GeV} / m_{\chi}\right)$ to bring their density to $\Omega_{\chi}=\Omega_{\mathrm{cdm}}$. This bound is derived from the BBN condition $T_{\mathrm{RH}} \gtrsim 5 \mathrm{MeV}$ by taking $\Omega_{\chi}=\Omega_{\mathrm{cdm}}$ in eq. (11). The condition $T_{\mathrm{RH}} \leq T_{\text {f.o. }}$ in Eq. (11) shows that for a solution $\Omega_{\chi}=\Omega_{\text {cdm }}$ with $\eta \lesssim 10^{-7}\left(100 \mathrm{GeV} / m_{\chi}\right)^{2}$ the production must be thermal with entropy dilution (case (1) or (2)), and $T_{\mathrm{RH}}$ must be close to $T_{\text {f.o. }}$. Notice that in between the two values of $\eta$ just mentioned the production is purely non-thermal (no chemical equilibrium, namely case (3)). For all overdense neutralinos, given one value of $\eta \lesssim 10^{-4}\left(100 \mathrm{GeV} / m_{\chi}\right)$ there is only one value of $T_{\mathrm{RH}}$ for which $\Omega_{\chi}=\Omega_{\mathrm{cdm}}$.

Underdense neutralinos, i.e. those with $\Omega_{\mathrm{std}}<\Omega_{\mathrm{cdm}}$, can have zero, one, or two solutions $\Omega_{\chi}=\Omega_{\mathrm{cdm}}$. There is no solution if $\Omega_{\text {std }}$ is too low. Neutralinos with $\Omega_{\text {std }} \lesssim 10^{-5}\left(100 \mathrm{GeV} / m_{\chi}\right)$ cannot be brought to $\Omega_{\mathrm{cdm}}$, independently of $\eta$. This can be seen by imposing the condition $T_{\mathrm{RH}}>5 \mathrm{MeV}$ and $\Omega_{\chi}=$ $\Omega_{\mathrm{cdm}}$ in Eq. (12). Neutralinos with $\Omega_{\mathrm{cdm}} \gtrsim \Omega_{\mathrm{std}} \gtrsim$ $10^{-5}\left(100 \mathrm{GeV} / m_{\chi}\right)$ cannot be brought to $\Omega_{\mathrm{cdm}}$ either if $\eta \lesssim 10^{-7}\left(100 \mathrm{GeV} / m_{\chi}\right)^{2}\left(\Omega_{\mathrm{cdm}} / \Omega_{\mathrm{std}}\right)$. This happens when the non-thermal production is insufficient to increase the density up to $\Omega_{\mathrm{cdm}}$, such as for the $\eta=10^{-7},\langle\sigma v\rangle=2 \times 10^{-7} \mathrm{GeV}^{-2}$ line in Fig. 1. Again using Eqs. (11) and (12) and Fig. 1, one can see that for the same range of densities $\Omega_{\text {cdm }} \gtrsim \Omega_{\text {std }} \gtrsim 10^{-5}\left(100 \mathrm{GeV} / m_{\chi}\right)$ there are two solutions for $10^{-7}\left(100 \mathrm{GeV} / m_{\chi}\right)^{2}\left(\Omega_{\mathrm{cdm}} / \Omega_{\mathrm{std}}\right) \lesssim \eta \lesssim$ $10^{-4}\left(100 \mathrm{GeV} / m_{\chi}\right)$ and a single solution for larger values of $\eta$. The two solutions have different values of $T_{\mathrm{RH}}$ and are both non-thermal one belonging to case (3) and the other to case (4). The single solution belongs to case (4).

In conclusion the neutralino density can be that of cold dark matter provided $\Omega_{\text {std }} \gtrsim 10^{-5}\left(100 \mathrm{GeV} / m_{\chi}\right)$ and the high energy theory accomodates the combinations of values of $b / m_{\phi}$ and $T_{\mathrm{RH}}$ identified in the previous two paragraphs. These conditions may place constraints on highenergy models derived from superstring theories.

This work was supported in part by the US Department of Energy Grant DE-FG03-91ER40662, Task C and NASA grants NAG5-13399 and ATP03-0000-0057 at UCLA, and NFS grant PHY-0456825 at the University of Utah. We thank A. Masiero, S. Nussinov, A. Soldatenko, and C. Yaguna for helpful discussions.
[1] D.N. Spergel et al. Astrophys. J., Suppl. Ser. 148, 175 (2003).

[2] T. Moroi and L. Randall, Nucl. Phys. B570, 455 (2000).

[3] M. Fujii, K. Hamaguchi, Phys. Rev. D 66, 083501 (2002); M. Fujii, M. Ibe, Phys. Rev. D 69, 035006 (2004).

[4] D. H. Lyth, E.D. Stewart, Phys. Rev. D 53, 1784 (1996).

[5] S. Hannestad, Phys. Rev. D 70, 043506 (2004).

[6] M. Kamionkowski, M. Turner, Phys. Rev. D 423310 (1990); R. Jeannerot, X. Zhang, R. Brandenberger, JHEP 12, 003 (1999); W. B. Lin, D. H. Huang, X. Zhang, R. Brandenberger, Phys. Rev. Lett. 86954 (2001).

[7] T. Moroi, M. Yamaguchi and T. Yanagida, Phys. Lett.B 342,105 (1995); M Kawasaki, T. Moroi and T. Yanagida, Phys. Lett.B 370,52 (1996).

[8] D. J.H. Chung, E. W. Kolb and A. Riotto, Phys. Rev. D60, 063504 (1999).

[9] G. F. Giudice, E. W. Kolb and S. Scopel, Phys. Rev. D64, 023508 (2001).
[10] R. Allahverdi and M. Drees, Phys. Rev. Lett. 89, 091302 (2002) and Phys. Rev. D66, 063513 (2002).

[11] S. Khalil, C. Muñoz and E. Torrente-Lujan, New Journal of Physics 4, 27 (2002); E. Torrente-Lujan, hep-ph/0210036 (2002).

[12] N. Fornengo, A. Riotto, Phys. Rev. D67, 023514 (2003).

[13] C. Pallis, Astrop. Phys. 21, 689 (2004).

[14] M. Endo, K. Hamaguchi, F. Takahashi, hep-ph/0602061 (2006); S. Nakamura, M. Yamaguchi, hep-ph/0602081 (2006).

[15] M. Hashimoto, K. I. Izawa, M. Yamaguchi, T. Yanagida, Prog. Theor. Phys. 100, 395 (1998); K. Kohri, M. Yamaguchi, J. Yokoyama, Phys. Rev. D 70, 043522 (2004); ibid. 72, 083510 (2005); J. Kaplan, hep-ph/0601262 (2006).

[16] J. McDonald, Phys. Rev. D 43 (1991) 1063. 